\title{
Ein Urbanismus der Ungleichheit
}

\author{
,Neue soziale Stadtpolitik' in Hamburg als Strategie der Verbürgerlichung
}

\section{Moritz Rinn}

In Deutschland werden gegenwärtig verschiedene stadtpolitische Re-Regulierungen beobachtet: neue Wohnungsbauprogramme, Instrumente zur Mietpreisbegrenzung oder Ansätze zur Restriktion von Investitionen in Gentrifizierungsgebieten. Nun wird diskutiert, ob solche ,sozialen' Re-Regulierungen einen Bruch mit urbaner Neoliberalisierung anzeigen. Der Aufsatz geht dieser Frage am Beispiel Hamburgs nach. Hier haben Forscher_innen kontroverse Positionen vertreten, ob ,progressive' Strategien hin zu einer ,Stadt für alle' zu beobachten sind. Der Aufsatz stellt zunächst die stadtpolitischen Re-Regulierungen vor und rekonstruiert anschließend die darin produktiven normativ-strategischen Rationalitäten politisch-administrativer Akteur_innen. Die zusammenführende Analyse zeigt, dass sich in Hamburg sozial-integrierte und unternehmerische Strategien zu einem ,Urbanismus der Ungleichheit' verdichten. Die ,Stadt für alle' soll durch Strategien der Verbürgerlichung realisiert werden, die Ungleichheiten verstärken.

Ersteinreichung: 18. Januar 2018; Veröffentlichung online: 20. Mai 2018

An english abstract can be found at the end of the document.

\section{Einleitung}

In der hiesigen Stadtforschung wird derzeit verstärkt diskutiert, wie jene stadtpolitischen Transformationen einzuschätzen seien, die in den letzten Jahren auf erstarkende städtische soziale Bewegungen und öffentliche Thematisierungen von Gentrifizierung, steigenden Mieten und,Wohnungsnot ${ }^{*}$ in zahlreichen deutschen Städten folgten. Politisch-administrative ReRegulierungen zielen vor allem auf eine Steigerung des Wohnungsneubaus in stark nachgefragten Städten und dabei auch auf die Schaffung von Sozialwohnungen. Einige Stadtregierungen setzen baurechtliche Instrumente zur Steuerung von Investitionen in Gentrifizierungsgebieten ein, um Verdrängungsprozessen entgegenzuwirken. Auf Bundesebene wurde versucht, Mietsteigerungen durch neue Rechtsinstrumente zu begrenzen. Nun steht die Frage im Raum, ob solche, sozial' orientierten Re-Regulierungen Anlass zur Hoffnung auf ein Ende urbaner Neoliberalisierung und auf eine gerechtere ,Stadt für alle' geben. Im vorliegenden Aufsatz diskutiere ich diese Frage am Beispiel der Stadt Hamburg. Hier haben etwa Vogelpohl und Buchholz (2017) zuletzt experimentelle Brüche mit vorherigen neoliberalen Stadtpolitiken beobachtet, während Metzger und Schipper (2017) stärker 
die fortgesetzte Markt- und Verwertungsorientierung wohnungspolitischer Re-Regulierungen betonen.

Hamburg eignet sich exemplarisch für die Diskussion gegenwärtiger stadtpolitischer Transformationen. Hier haben städtische soziale Bewegungen in der jüngeren Vergangenheit machtvoll Konflikte um die ,Stadt für alle' artikuliert, die ebenso wie die folgenden Reaktionen politischadministrativer Akteur_innen in der Stadtforschung breit diskutiert wurden (vgl. u.a. Metzger/Schipper 2017, Vogelpohl/Buchholz 2017, Rinn 2016, Vrenegor 2012, Füllner/Templin 2011, Birke 2010). Im folgenden zweiten Abschnitt stelle ich die teils kontroversen Analysen dieser ,neuen sozialen Stadtpolitik' in Hamburg zunächst in den Kontext wohnungspolitischer Transformationen auf den verschiedenen politisch-administrativen Ebenen der Bundesrepublik. Um am Hamburger Beispiel nun mögliche stadt- und wohnungspolitische Brüche beziehungsweise Kontinuitäten herausarbeiten zu können, schlage ich im dritten Abschnitt eine genealogisch und staatsanalytisch orientierte Analyseperspektive auf das stadtentwicklungspolitische Machtfeld vor. Denn, so mein Argument, die gegenwärtigen - vor allem wohnungspolitischen - Re-Regulierungen können analytisch schärfer eingeordnet werden, wenn die stadtpolitischen Rationalitäten und Institutionalisierungen rekonstruiert werden, die in diesen Re-Regulierungen produktiv werden und diese in ihren normativstrategischen Ausrichtungen prägen. Stadtpolitische Rationalitäten und Institutionalisierungen werden dabei in genealogischer Perspektive als ,Produkte' von Konflikten um die Produktion und Aneignung von Stadt begriffen und Stadtpolitik zugleich als Element demokratisch-kapitalistischer Staatlichkeit verortet, das heißt als Teil eines Ensembles gesellschaftlicher Konfliktregulation und Stabilisierung eines Krisenzusammenhangs.

Mit Hilfe dieser analytischen Perspektive rekonstruiere ich im vierten Abschnitt die dominanten politisch-administrativen Strategien städtischer Regierung in Hamburg, die sich historisch ausgehend von der Krise der fordistischen Stadt in den 1970er Jahren herausgebildet haben, und reinterpretiere vor diesem Hintergrund in Abschnitt fünf die gegenwärtigen politisch-administrativen Re-Regulierungen.[1] Ich komme dabei zu dem Ergebnis, dass in den diskutierten stadtpolitischen Transformationen kaum potenziell progressive oder positiv als ,sozialer' verstandene Veränderungen sichtbar werden. Die empirische Analyse zeigt vielmehr, dass die beobachteten ,sozialeren' Politiken nicht in einer einfachen Opposition zu unternehmerisch-neoliberalen stehen, sondern dass beide zentrale, historisch gewordene normativ-strategische Grundlagen teilen, die einen ,Urbanismus der Ungleichheit‘ konstituieren. Sichtbar wird eine dominante Rationalität unternehmerisch-integrierter Stadtentwicklungspolitik, die auf ökonomisch dynamische Entwicklung und soziale Kohäsion der Gesamtstadt und ihrer Teilräume abzielt und dabei vor allem mit quartiersbasierten, mittelschichtsorientierten Normalisierungsstrategien und einer Bevölkerungspolitik der ,sozialen Mischung' arbeitet. Das politisch-administrative Versprechen einer ,Stadt für alle' soll so maßgeblich durch Strategien der ,Verbürgerlichung' städtischer Räume eingelöst werden, die soziale Ungleichheiten verstärken anstatt ihnen entgegenzuwirken. 


\section{Gegenwärtige wohnungspolitische Transformationen in Deutschland und das Hamburger Beispiel}

In den gegenwärtig in Deutschland zu beobachtenden stadtpolitischen Veränderungen wird einmal mehr sichtbar, welche Effekte städtische soziale Bewegungen auf Stadtentwicklungspolitik haben können. Denn einer der Ausgangspunkte dieser Transformationen waren die Interventionen von Initiativen und Netzwerken, die sich ab Ende der 2000er Jahre in zahlreichen Großstädten - Hamburg, Berlin, Frankfurt, Düsseldorf, Leipzig oder Jena - bildeten. Diese richteten sich vor allem gegen Gentrifizierung, steigende Mieten, Zwangsräumungen und Verdrängung und forderten insgesamt eine andere Wohnungspolitik sowie partizipative bis selbstorganisierte Formen der Stadtplanung (vgl. Vogelpohl et al. 2017). Ihre Interventionen öffneten ein Aufmerksamkeitsfenster, in dem etwa die öffentliche Verantwortung für sozialen Wohnungsbau wieder verstärkt thematisiert werden konnte (vgl. Schönig et al. 2017, Metzger/Schipper 2017). Es folgten politischadministrative Reaktionen, welche die überschießenden Forderungen nach egalitärer Aneignung städtischer Räume und Vergesellschaftung städtischer Produktionsweisen den normativen wie strategischen Selektivitäten staatlicher Stadtpolitik entsprechend be- und verarbeiteten.

In den Untersuchungen dieser Transformationen dominieren bislang vergleichende Diskussionen von Fallstudien zu einzelnen Städten, insbesondere hinsichtlich der Wohnungspolitik (vgl. etwa Holm 2017, Metzger/ Schipper 2017, Schönig etal.2017, Vogelpohl/Buchholz 2017). Vor dem Hintergrund höchst ungleicher Entwicklungen von städtischen Bevölkerungen und Wohnungsmärkten sowie der dezentralen föderalen Verfasstheit der Bundesrepublik, in deren Rahmen den Bundesländern und gerade der kommunalen Ebene in den letzten Jahren sukzessiv mehr Verantwortung für die Wohnraumversorgung übertragen wurde (vgl. Kaltenbrunner/Waltersbacher 2014), liegt es nahe, zunächst von Stadtpolitiken im Plural zu sprechen. Dennoch lassen sich übergreifende Tendenzen stadtpolitischer Transformationen in jenen deutschen Städten identifizieren, in denen eine neue ,Wohnungsnot' als zu bearbeitendes Problem politisch-administrativ anerkannt wurde: Auf kommunaler Ebene (bzw. in den Stadtstaaten Berlin, Hamburg und Bremen) werden Bündnisse zwischen politisch-administrativen Akteur_innen und der Wohnungswirtschaft mit dem Ziel gebildet, den Neubau von (teils öffentlich geförderten) Wohnungen zu steigern. Daneben wird hier vereinzelt das städtebaurechtliche Instrument der ,Sozialen Erhaltungssatzung' zur Regulation von Investitionen in Gentrifizierungsgebieten eingesetzt. Noch vereinzelter und eher prospektiv werden veränderte Bewirtschaftungsstrategien öffentlicher Wohnungsbaugesellschaften, also eine Abkehr von gewinnorientierten hin zu mieter_innenfreundlicheren Strategien beobachtet - oder zumindest die Diskussion dessen (etwa in Berlin oder Frankfurt, vgl. Holm 2017, Metzger/Schipper 2017, Schönig et al. 2017). Aber auch auf der Länder- und Bundesebene wurde wohnungspolitisch reagiert und mittlerweile (wieder) eine Notwendigkeit anerkannt, stärker steuernd auf den Wohnungsmarkt einzuwirken. Dafür wurde der im Rahmen der Föderalismusreform beschlossene Ausstieg des Bundes aus der sozialen Wohnungsbauförderung verschoben und die Mittel für soziale 
Wohnraumversorgung im Bereich der Objekt- wie der Subjektförderung erhöht. Mit der - allerdings stark kritisierten und durch widerstreitende Rechtsprechungen in der Wirkung zusätzlich begrenzten - ,Mietpreisbremse wurde ein Instrument eingeführt, das in von den Landesregierungen definierten Gebieten „mit einem angespannten Wohnungsmarkt“ (§ 556d (1) BGB) dämpfend auf Mieterhöhungen bei Neuvermietungen wirken soll (vgl. zur Kritik Holm 2014).

Wenn der Blick nun auf Hamburg gerichtet wird, dann scheinen die dortigen neuen wohnungspolitischen Programmatiken und Instrumente zum bundesweiten Mainstream, wenn nicht gar zu den strategischen Trendsettern zu gehören. In der zweitgrößten Stadt Deutschlands, die durch deutlich sichtbare verräumlichte soziale Ungleichheiten gekennzeichnet ist (vgl. Breckner 2013, Güntner 2013) und deren Bevölkerungszahl seit Jahren kontinuierlich steigt, stellten politisch-administrative Bemühungen um eine ,Stadt für alle zunächst eine Reaktion auf die Konfliktartikulationen städtischer sozialer Bewegungen dar. Denn spätestens mit der Gründung des „Netzwerks Recht auf Stadt“ im Jahr 2009 wurde eine breitere Unzufriedenheit mit städtischen Lebensverhältnissen in der Hansestadt überdeutlich, die auch von Teilen der ,Mittelschicht ${ }^{\text {- }}$ dem Kernklientel der regierenden Parteien - geteilt wurde. Neben den als ,Proteste der Kreativen“ begriffenen Arbeitskonflikten und stadtpolitischen Interventionen von (vorwiegend prekären) Kulturschaffenden organisierten sich Initiativen vornehmlich in den zentrumsnahen Stadtteilen gegen Gentrifizierung und Mietsteigerungen[2], hochpreisigen Wohnungsneubau oder die Zerstörung städtischer Grünflächen (vgl. Fraeser 2017, Vrenegor 2012, Füllner/Templin 2011, Birke 2010). Verbindende Elemente waren dabei die egalitäre Forderung nach einer ,Stadt für alle“ und der protagonistische Anspruch auf eine „Stadtplanung von unten“ (Füllner/Templin 2011, vgl. Rinn 2016). Durch ein breites Repertoire von Interventionen gelang es dem Netzwerk nicht nur eigene Projekte durchzusetzen und Planungen von ImmobilienVerwertungs-Koalitionen zu verhindern oder zumindest zu verändern - es gelang vielmehr, die stadtpolitischen Kräfteverhältnisse zumindest temporär und lokal zu verschieben.

Diese intensivierten Konflikte sind nicht an der akademischen Stadtforschung vorbeigegangen. Diskutiert wurden dabei auch die strategischen Reaktionen politisch-administrativer Akteur_innen der Stadtplanung und Stadtentwicklungspolitik und insofern die Effekte der Bewegungen auf staatliche Politiken. Denn angesichts der neuen Mobilisierungsdynamik versuchten politisch-administrative Akteur_innen, die Deutungshoheit über Probleme der Stadtentwicklung und deren Lösungen zurückzuerlangen. In der Hochphase der „Recht auf Stadt"-Mobilisierung wurde Hamburg durch die CDU und die Grün-Alternative Liste regiert, wobei letztere 2011 die Koalition aufkündigte. Im Vorfeld, während des Wahlkampfs und im Anschluss an die Neuwahlen zur Bürgerschaft, aus der die SPD mit absoluter Mehrheit hervorging, waren Stadtentwicklung und Wohnen die zentralen politischen Themen - die SPD warb explizit mit dem Versprechen einer „Stadt für alle“ (Rinn 2016: 187; vgl. Schubert 2011).

Für dienun folgendestadt-und wohnungspolitische Rekonfigurationsphase wurden maßgeblich drei Verschiebungen beobachtet: erstens die Ausweitung 
des öffentlich subventionierten sozialen Wohnungsbaus im Rahmen eines Neubauprogramms. Pro Jahr sollten zunächst 6.00o, seit 201610.000 neue Wohnungen gebaut werden, wobei nach der sogenannten ,Drittelmix-Regelung' zu je einem Drittel sozial geförderte Mietwohnungen, frei finanzierte Mietwohnungen und Eigentumswohnungen vorgesehen sind (vgl. Metzger/ Schipper 2017, Vogelpohl/Buchholz 2017).[3] An der Realisierung dieser Ziele soll das 2011 initiierte „Bündnis für das Wohnen“ aus Wohnungswirtschaft, Kommunalpolitik und -verwaltung sowie Mieterverbänden gemeinsam arbeiten. Parallel wurde ein stärker auf,mittlere Einkommensgruppen' ausgerichteter Förderweg mit einer höheren Anfangsmiete als im ,normalen“ Sozialwohnungsbau eingerichtet (2017 betrugen die Kaltmieten 6,40 bzw. 8,50 Euro pro $\mathrm{m}^{2}$ ). Wohnungsneubau war also die zentrale Strategie des Senats gegen steigende Mieten. In diesem Kontext steht auch die Umstellung der Vermarktung stadteigener Grundstücke von einem Höchstgebotsverfahren hin zur Konzeptvergabe, welche an den neuen wohnungsbaupolitischen Zielen orientiert sein soll (vgl. Rinn 2016: 182ff.).

Zweitens wurde der verstärkte Einsatz der schon erwähnten baurechtlichen Restriktionen für Bestandsinvestitionen in Gentrifizierungsgebieten beobachtet. In Hamburg sind es „Soziale Erhaltungsverordnungen“, mit denen Luxussanierungen und Umwandlungen von Miet- in Eigentumswohnung in von Verdrängungsprozessen bedrohten Gebieten unter einen besonderen Genehmigungsvorbehalt gestellt werden können. Dieses Instrument hat allerdings isoliert nur beschränkte Wirkungen auf allgemeine Mietsteigerungsdynamiken (vgl. Vogelpohl 2013). Nahezu alle der acht seit 2012 beschlossenen Gebiete liegen in der westlichen inneren Stadt und angrenzenden Teilen von Ottensen und Eimsbüttel und waren zuvor Sanierungsgebiete oder Fördergebiete des Programms „Soziale Stadt“ (vgl. auch FHH 2017d, Rinn 2016: 187).

Darüber hinaus wurden nun drittens auch Veränderungen von Entwicklungsstrategien für ,benachteiligte“ Stadtteile festgestellt. In ihrer Analyse der neuen Stadtpolitiken in Hamburg verweisen Vogelpohl und Buchholz (2017) prominent auf die Internationale Bauausstellung (IBA), die zwischen 2006 und 2013 in den traditionell proletarisch und durch Migration geprägten Stadtteilen Wilhelmsburg und Veddel durchgeführt wurde. In diesen jahrzehntelang als ,Problemquartiere' behandelten Gebieten wurde durch eine stadteigene Entwicklungsgesellschaft an einer umfassenden symbolischen, ökonomischen und bevölkerungsbezogenen Aufwertung gearbeitet und insgesamt rund eine Milliarde Euro an privaten Investitionen in Immobilienprojekte und Infrastrukturen mobilisiert (vgl. Eckardt 2017, Birke/Hohenstatt/Rinn 2015, AKU Wilhelmsburg 2013, Breckner 2013, Hohenstatt/Rinn 2013). Dagegen organisierten sich lokale Stadtteilinitiativen und kritisierten die IBA als Instrument staatlich vorangetriebener Gentrifizierung. Die IBA habe nun, so Vogelpohl und Buchholz, auf diese Kritiken reagiert, eine „Aufwertung ohne Verdrängung“ versprochen und dieses Versprechen wohnungsbaupolitisch mit einem beispielhaften Modellprojekt der Bestandssanierung einzulösen versucht (Vogelpohl/ Buchholz 2017:270ff.). Dieses Projekt namens „Weltquartier“ habegezeigt, wie eine umfassende Modernisierung sanierungsbedürftiger Bestandswohnungen des städtischen Wohnungsunternehmens SAGA mit verlängerten Laufzeiten 
des sozialen Wohnungsbaus kombiniert werden könne, sodass statt den üblichen 15 nun 30 Jahre lang, soziale' Mieten garantiert werden. Diese Interpretation der Reaktion der IBA auf Gentrifizierungskritik und gerade ihre Einordnung in die neuen,sozialen' Re-Regulierungen Hamburger Stadtentwicklungspolitik ist tatsächlich überraschend, bisherige Analysen (auf die ich im fünften Abschnitt dezidiert eingehen werde) hatten vor allem die mittelschichtsorientierten Aufwertungsstrategien und entsprechende Verdrängungseffekte hervorgehoben.

Wie werden diese hier nur knapp skizzierten Transformationen nun insgesamt in ihrer Reichweite eingeschätzt? Die Untersuchungen von Schönig et al. (2017) zu Berlin, München, Dresden, Leipzig und Hannover sowie von Metzger und Schipper (2017) zu Hamburg und Frankfurt kommen zu skeptischen Ergebnissen: Auch wenn im Vergleich zu den ,neoliberalen“ 20ooer Jahren Unterschiede vor allem der kommunalen Wohnungspolitiken deutlich würden (wie etwa neue Wohnungsbaukonzepte, die Forderung nach mehr Sozialwohnungen, eine breit geteilte Kritik an Privatisierungen kommunaler Wohnungsbestände sowie deren partielle Rekommunalisierung), so seien klare Brüche mit einer primär marktförmigen Organisation der Wohnraumversorgung oder eine paradigmatische „Um- oder Rückkehr zu wohlfahrtsstaatlicher Wohnungspolitik“ (Schönig et al. 2017: 26) bislang kaum erkennbar. Auch ,post-neoliberale‘ Entwicklungen, verstanden als Dekommodifizierung und Demokratisierung der Stadt- beziehungsweise Wohnungspolitik, ließen sich nicht ausmachen (vgl. Metzger/ Schipper 2017). Im Unterschied dazu kommen Vogelpohl und Buchholz für Hamburg allerdings zu dem Ergebnis, es handele sich bei den neuen wohnungsbaupolitischen Instrumenten, den Sozialen Erhaltungsverordnungen und dem veränderten Ansatz der IBA Hamburg um „Experimente, um Verdrängung [,property-led displacement'] zu reduzieren, den Markt zu re-regulieren und preisgebundenen Sozialwohnungsbau zu stärken. Diese Experimente sind wichtige Schritte in Richtung einer Stadt für alle“ (Vogelpohl/Buchholz 2017: 278f., Übers. d.A.). Obgleich die Autor_innen die Grenzen dieser „Experimente“ durchaus diskutieren, interpretieren sie die wohnungspolitischen Re-Regulierungen insgesamt als Ansätze, die darauf abzielten, die „neoliberale Stadt zu begrenzen“ (ebd.: 266), und identifizieren sogar mögliche Wendepunkte hin zu einer progressiveren Stadtpolitik.

Am Hamburger Beispiel zeichnet sich also offenbar eine Kontroverse ab. Dabei ist zunächst kaum bestreitbar, dass programmatische Anstrengungen politisch-administrativer Akteur_innen zu beobachten waren, die explizit mehr ,bezahlbaren' Wohnraum und eine Wohnungspolitik ,für alle، versprachen (vgl. dazu auch Rinn 2016: 182ff.). Worin aber besteht dieses Versprechen, Hamburg als ,Stadt für alle zu entwickeln, und wie soll diese Stadt durch die beschrieben Strategien und Instrumentarien hervorgebracht werden? Diese Fragen verweisen auf diegrundierenden stadtpolitischen Rationalitäten, die in den kontrovers diskutierten Re-Regulierungen produktiv werden. Um mögliche Kontinuitäten oder Brüche gegenwärtiger Stadtpolitik in Hamburg herausarbeiten zu können, halte ich es deshalb zunächst für notwendig, diese Rationalitäten genauer zu analysieren. Es gilt, sie in ihrer geschichtlichen Herausbildung sowie institutionellen Einbettung in ein Ensemble demokratisch-kapitalistischer Staatlichkeit zu rekonstruieren. Auf 
dieser Grundlage kann anschließend das gegenwärtige Verhältnis ,sozialer und ,neoliberaler' Stadtpolitiken in Hamburg bestimmt und die,neue soziale Stadtpolitik analytisch eingeordnet werden. Im folgenden Abschnitt stelle ich nun zunächst die entsprechende genealogische und staatsanalytisch informierte Untersuchungsperspektive auf stadtentwicklungspolitische Transformationen vor (vgl. auch Rinn 2016: 26ff.).

\section{Genealogische und staatsanalytische Perspektiven auf stadtpolitische Transformationen}

Zentraler analytischer Ansatzpunkt der hier eingenommenen Perspektive auf Stadtentwicklungspolitiken und ihre Transformationen sind Konflikte um die Hervorbringung, das heißt die Produktion, Aneignung und Nutzung städtischer Räume. Die Analyse setzt an konkreten Konstellationen und Praktiken konfliktbeteiligter Akteur_innen an. Alle diese Akteur_innen - wie etwa städtische Planungsabteilungen, Investor_innen, zivilgesellschaftliche Organisationen oder städtische soziale Bewegungen - agieren auf Basis von je spezifischen, historisch gewordenen normativen wie strategischen Wissens- und Praxisrepertoires und damit spezifischer institutionalisierter Machtressourcen. Diese sind als relational zu begreifen, sie werden innerhalb konflikthafter Beziehungen praktisch produktiv und dabei zugleich re-artikuliert und transformiert. Um nun die normativ-strategischen Positionierungen der hier im Fokus stehenden politisch-administrativen Akteur_innen analysieren zu können, müssen diese als Teil eines spezifisch materialisierten Ensembles von Staatlichkeit begriffen werden. Historisch haben sich Stadtentwicklungspolitik und Stadtplanung als Elemente moderner, kapitalistischer (Wohlfahrts-)Staatlichkeit und spezifischer patriarchaler Machtbeziehungen konstituiert (vgl. Häußermann/Läpple/Siebel 2008, Frank 2003). Sie sind insofern Instrumentarium der sozialen Disziplinierung aber auch repressiver Klassenpolitik (vgl. Holm 2006: 25ff.). Gleichzeitig wird in ihnen eine gouvernementale Regierungsrationalität produktiv (vgl. Foucault 2004): eine Bevölkerungspolitik, die auf die,rationale‘ Optimierung der Zusammensetzung, räumlichen Anordnung und Entwicklungsdynamik der jeweiligen städtischen Bevölkerung und ihrer spezifisch verräumlichten Segmente gerichtet ist. Dieser Bevölkerungspolitik geht es um die Herstellung und Absicherung der Re-Produktionsbedingungen ,bürgerlicher Vergesellschaftungsformen (im ökonomischen, politisch-sozialen und kulturellen Sinne, vgl. Lanz 2013). Stadtentwicklungspolitik wird also als Politik des Sozialen gefasst, die zugleich Kohäsion und dynamische Entwicklung der bürgerlichen Gesellschaft im städtischen Raum organisieren soll. Damit wird sie als Element gegenwärtiger demokratisch-kapitalistischer Staatlichkeit konzipiert, durch die hindurch gesellschaftliche Konflikte und Krisen reguliert und zu stabilisieren versucht werden (vgl. Lessenich 2008). Dieses Ensemble von Staatlichkeit ist durch spezifische Selektivitäten charakterisiert, die es unterschiedlichen nicht-staatlichen Akteur_innen auf verschiedene Arten und Weisen ermöglichen, ihre partikularen Interessen und Strategien mittels Zugang zu staatlichen Ressourcen oder durch deren Kontrolle durchzusetzen (vgl. Jessop 2002: 40). Das wird gerade in der dominanten ,Mittelschichtsorientierung ${ }^{6}$ von gegenwärtiger Stadt- und Wohnungspolitik 
sichtbar, die auch als Arbeit an einer, Verbürgerlichung'[4] der Stadt begriffen werden kann. Dabei beanspruchen politisch-administrative Akteur_innen jedoch als staatliche Kernkompetenz, das Gemeinwohl herzustellen, das die Partikularinteressen in sich aufhebt. Das Gemeinwohl bleibt dabei als Legitimationsressource politisch-administrativer Akteur_innen immer umstritten - es ist konstitutiv unvollständig (da einige Interessen privilegiert, andere ausgeschlossen werden) und so imaginär wie die Gemeinschaft, deren allgemeiner Willen in ihm repräsentiert sein soll (vgl. Jessop 2007: 9ff., Demirovic 2013). Die diskursive Figur des Gemeinwohls ist damit Gegenstand dauerhafter Konflikte - in den Debatten um die gegenwärtigen Stadtentwicklungspolitiken in Hamburg geht es dabei um die, gute Stadt ' und auch die ,Stadt für alle' (vgl. Rinn 2016).

Für die Untersuchung der Transformationen Hamburger Stadtentwicklungspolitik schlage ich also vor, städtische Krisen, Konflikte um Stadtentwicklung und um ihre Politiken zum Ausgangspunkt einer genealogischen Rekonstruktion zu machen. Mit einem solchen analytischen Ansatz kann auch die Frage nach Brüchen oder Kontinuitäten der gegenwärtigen, kontrovers diskutierten Stadtpolitiken historisch fundiert diskutiert werden. Dabei wird die folgende Analyse sichtbar machen, wie in diesen stadtpolitischen Re-Regulierungen zwei verschiedene Stränge der Bearbeitung städtischer Krisen konvergieren: ,soziale‘, integrierte Strategien der Entwicklung ,problematischer ' Quartiere sowie unternehmerische Stadtpolitik. Bevor ich die gegenwärtigen Re-Regulierungen im fünften Abschnitt re-interpretiere, gilt es daher zunächst, diese sozial-integrierten wie unternehmerischen Stadtpolitiken genauer zu analysieren. Beide adressieren die Krise der fordistisch-funktionalen Stadt, die sich in Hamburg ab Ende der 1960er Jahre abzeichnete und die im Verlauf der 1970er Jahre zur vollen Entfaltung kommen sollte.

\section{Die Herausbildung sozial-integrierter und unternehme- rischer Krisenbearbeitungsstrategien in Hamburg}

Im Rückblick auf die Stadtkrise der 1970er Jahre tritt ein Bruch stadtentwicklungspolitischer Rationalitäten deutlich zu Tage: Noch 1969 veröffentlichte die Hamburger Baubehörde ein zukunftsweisendes „Entwicklungsmodell“, das eine konsequent funktionalistische Restrukturierung der Stadt vorsah, wobei ein Großteil der innerstädtischen Altbaugebiete mit Dienstleistungs-, Management- und Verkehrsnutzungen ,überplant wurde. Präferiertes Wohnungsbaumodell war die Großwohnsiedlung. Diese funktionale Umstrukturierung scheiterte jedoch an gleich mehreren Faktoren. Einerseits geriet sie mit der Krise der fordistischen Gesellschaftsformation in Konflikt (vgl. Rinn 2016: 77ff.): Sinkende Steuereinnahmen, sozial selektive Bevölkerungsverluste und wachsende Staatsausgaben (etwa für Sozialtransfers, Schuldendienst und die Subventionierung niedergehender Wirtschaftsbranchen) führten zu einer Krise des städtischen Haushaltes, die die ambitionierten Planungen stoppte. Gleichzeitig stieß sie auch bei den Bewohner_innen auf Widerstand. Die Planung peripherer Großwohnsiedlungen war verkoppelt mit dem spekulativen Herunterwirtschaften der zum Abriss freigegebenen innenstadtnahen Wohngebiete. 
Dort organisierten sich Stadtteilinitiativen gegen Kahlschlagsanierung und ,Umsiedlung‘. Der Suburbanisierungstrend schließlich, in dem vor allem überdurchschnittlich verdienende Bevölkerungsgruppen ihr Eigenheim im Grünen realisierten (vgl. Häußermann/Läpple/Siebel 2008: 87), trug mit zur Neuzusammensetzung der Bewohner_innenschaft der Innenstadtgebiete bei - und aus der Perspektive politisch-administrativer Akteur_innen zu einer veritablen Krise des städtischen Lebensmodells, da sich nun vor allem Erwerbslose, Arme, Arbeitsmigrant_innen und ihre Familien in den zentrumsnahen Stadtteilen ,konzentrierten'.

Die hier anklingende Problemdiagnose hinsichtlich der inneren Stadt wie der Großwohnsiedlungen wurde ab Ende der 1970er Jahre bundesweit im Diskurs um „soziale Brennpunkte“ produktiv. In Hamburg tauchte dieser Begriff konzeptuell erstmals im „Stadtentwicklungskonzept“ von 1980 auf (FHH 1980). In diesem Zusammenhang wurde das Quartier, begriffen als spezifisch bevölkerter und bewirtschafteter Teil-Raum der Stadt, als zentrale Planungs- und Steuerungseinheit für eine erneuerte städtische Sozialpolitik ,entdeckt' (vgl. Rinn 2016: 88ff.). Teil dieser Entdeckung war die Produktion von Wissen über problematische oder wünschenswerte Bevölkerungszusammensetzungen von Quartieren hinsichtlich sozialer Integration. Gleichzeitig verschoben sich stadtgesellschaftliche Problem- und Zieldefinitionen von der (nie eingelösten) Vorstellung, soziale Ungleichheit müsste durch einen sukzessiven Aufstieg aller Bewohner_innen in Mittelschichtspositionen abgebaut werden, hin zur Vermeidung „homogener“ Bevölkerungsstrukturen - gemeint war eine Konzentration armer, proletarischer und migrantisch positionierter Bewohner_innen - in bestimmten ,gefährdeten Stadtteilen“ (FHH 1980, vgl. auch Alisch/Dangschat 1998: 122), womit die „sozialen Gegensätze der Stadt“ entschärft werden sollten (vgl. Bauriedl 2007: 96f). Auf die Diagnose verräumlichter Desintegration wurde dabei folgerichtig mit ebenfalls verräumlichten Strategien reagiert: Durch die Ausweitung des Angebotes von suburban anmutenden Siedlungsformen innerhalb des Stadtgebietes und durch die Attraktivierung der bestehenden innerstädtischen Wohngebiete sollte die Stadt wieder zur Wohnoption für ,steuerkräftige‘ Bewohner_innen und ,sozial stabile Familien“ werden (vgl. die administrative Perspektive bei Polkowski 2001). Parallel zu dieser ,sozial-integrativen“ Konzeption bildete sich nun verstärkt ab Mitte der 1980er Jahre eine ,integrierte'Stadtteilentwicklung mit ressortübergreifenden und kooperativ-partizipativen Steuerungsformen heraus (vgl. Rinn 2016: 113ff.). In einem konflikthaften Institutionalisierungsprozess, in den auch Teile der entstandenen ,alternativen Stadterneuerungsszene eingebunden wurden, entwickelte sich ein erneuertes Stadtentwicklungsregime. Dieses war unter anderem darauf orientiert, das Leben in der Innenstadt für eine als ausdifferenziert und in ihren Lebensstilen pluralisiert begriffene Mittelschicht attraktiv zu machen, wobei eine dichte, kleinteilige und spezifisch innerstädtische,Urbanität‘ mit aktivem bürgerschaftlichem Engagement verkoppelt wurde.

In den 1990er Jahren schließlich wurde die quartiersorientierte Integrationspolitik als die soziale Stadtpolitik normalisiert und verallgemeinert und auch auf der Senatsebene durch die Gründung einer Stadtentwicklungsbehörde institutionalisiert. Die Herausbildung dieses Regimes der integrierten Stadtteilentwicklung vollzog sich dabei in Korrespondenz 
mit entsprechenden Programmen auf EU- und Bundesebene (vgl. dazu Güntner 2007). Das Wissens- und Praxisrepertoire behutsamer Stadtteilentwicklung unter aktiver Beteiligung der Bewohner_innen und Nutzung ihrer Selbsthilfepotenziale ermöglichte jene neuen, verräumlichten Ansätze der Bekämpfung von Armut als ,soziale Exklusion“ (vgl. Mayer 2002). Der normativ-strategische Kern dieser sozial-integrierten Stadtteilentwicklung lässt sich wie folgt zusammenfassen (vgl. Rinn 2016: 126ff.): Städtische Armut wurde als Desintegrationsproblem begriffen, das sich vor allem in peripheren Großwohnsiedlungen, aber auch noch in innenstadtnahen Altbaugebieten konzentriere. Diese befänden sich in einer selbstverstärkenden Abwärtsspirale: Durch die ,Flucht der Mittelschichten“ verschlechtere sich die Lebensqualität etwa durch die zunehmende Erosion von ökonomischen, sozialen und Bildungsinfrastrukturen - mit der Folge weiterer Fortzüge. Übrig blieben ,benachteiligte Bevölkerungsgruppen', deren Wohnviertel zu einem zusätzlich benachteiligenden Faktor würden. Sozialisationsdefizite und Alltagsdevianz, ,Sozialhilfeabhängigkeit‘ und physisch-räumliche wie soziale,Verwahrlosung 'würden sich potenzieren, wenn in der unmittelbaren Nachbarschaft jene Bewohner_innen fehlten, welche die Normen bildungsund lohnarbeitsorientierter, ,westlicher bürgerlicher Lebensweisen repräsentieren könnten - Bewohner_innen, die sich auch für bessere Lebensbedingungen und Investitionen in ihre Quartiere einsetzen könnten, da sie im politischen Raum Gehör fänden: ,junge Kreative‘, ,Familien mit stabilen Einkommen', ,sozial engagierte Mittelschichten'. Den ,Problemquartieren“ wurde damit ein Mangel an Bürgerlichkeit attestiert (vgl. etwa der damalige Stadtentwicklungssenator Maier 1999: 14).[5] Als Kernproblem städtischer Armut wurden - und hier folgte Hamburg geradezu prototypisch den international beobachteten stadtpolitischen Trends - negative Kontexteffekte ausgemacht, die mit Politiken sozialer Mischung zu bearbeiten seien (vgl. dazu Huning/Schuster 2015). Eine mittelschichtsorientierte Aufwertung innerstädtischer ,Problemquartiere - in den Altbauquartieren der westlichen inneren Stadt also der Erhalt historischer Bausubstanz und die Verbesserung von Stadtteilimage, Wohnumfeld und Bildungsinfrastrukturen - wurde damit zur expliziten Strategie gegen soziale Ausgrenzung. Die integrierte soziale Stadtteilentwicklungspolitik bedeutete damit de facto staatlich initiierte Gentrifizierung.

Den zweiten Strang der Bearbeitung städtischer Krisen seit dem Bruch der 1970er Jahre bilden nun dezidiert ökonomische beziehungsweise unternehmerische Strategien. Programmatischer Wendepunkt war hier ohne Zweifel die mit „Unternehmen Hamburg“ betitelte Rede des Ersten Bürgermeisters Klaus von Dohnanyi im Jahr 1983 (Dohnanyi 1983). Dohnanyi kündigte in dieser Rede einen wirtschaftspolitischen Paradigmenwechsel an: Einen Bruch mit der (zuvor gescheiterten) Strategie, niedergehende hafenbezogene Industriezweige zu subventionieren und weitere Industrialisierungsprojekte zu entwickeln, und eine aktivere Wirtschaftsförderungspolitik, die sich vor allem auf Kommunikations- und IT-Dienstleistungen, den Ausbau des Medien- und Messestandortes sowie die Anwerbung wissenschafts- und technikorientierter Industrien konzentrierte (vgl. Dangschat 1993). Stadtund sozialpolitisch forderte Dohnanyi die „Entwicklung von Wohnqualität, Freizeitwert und Kultur, ausgerichtet am Geschmack einer ,neuen Intelligenz', 
,die Schöpfer der neuen Industrien und Dienstleistungen“ ist“ (Alisch/ Dangschat 1998: 114f.). Die unternehmerische Programmatik und Strategie prägte in den Folgejahrzehnten den stadtentwicklungspolitischen Kurs Hamburgs. Die Produktion ,repräsentativer Räume' und attraktiver Quartiere für die umworbenen hochqualifizierten Arbeitskräfte wie (Einkaufs-) Tourist_innen stand auf der Tagesordnung und motivierte auch Kontrollund Vertreibungsstrategien gegenüber ,Randgruppen ( oder Wohnungslosen, vgl. Peddinghaus/Hauer 1998). Ab Mitte der 1990er Jahre wurde mit der HafenCity ein neuer Stadtteil auf city-nahen ehemaligen Hafenarealen geplant, der für seine ,komplexe urbanistische Konzeption gefeiert wurde (vgl. etwa Lees 2010) - dabei aber vor allem für öffentliche Subventionierungen spektakulärer Neubauprojekte, internationale Unternehmensansiedlungen, hochpreisigen Wohnungsbau und hochwertige Shopping-Areale steht (vgl. Bauriedl 2007). Als die 2001 gebildete Senatskoalition aus CDU, FDP und der rechtspopulistischen Schill-Partei ihr Leitbild „Metropole Hamburg - Wachsende Stadt“ präsentierte, wurde neoliberale Stadtpolitik schließlich in Reinform forciert. Das bevölkerungs- wie wirtschaftspolitische Wachstumsprogramm und die entsprechenden städtebaulichen Aufwertungsstrategien zielten ausschließlich auf die Steigerung der Konkurrenzfähigkeit bei der Anwerbung von Unternehmen, Tourist_innen und hochqualifizierten ,jungen kreativen Menschen“ (FHH 2002: 13), während explizit sozialpolitische Ansätze völlig verschwanden. Die neue Senatskoalition arbeitete konsequent mit Marketingstrategien und spektakulärem Städtebau, insbesondere das Konzerthaus Elbphilharmonie ab 2006 steht für eine Stadt- und Kulturpolitik, die auf den ,Bilbao-Effekt‘ zielt und dabei ihre ,Zukunftsprojekte durch Einsparungen, Privatisierungen und den aus Mieterhöhungen erzielten Gewinnen der stadteigenen Wohnungsbauunternehmen finanzierte (vgl. Birke 2010). Teil dieser intensivierten neoliberalen Stadtpolitik war auch die Inwertsetzung ,untergenutzter städtischer Räume als Wohnbau- und Gewerbeflächen sowie die Vermarktung städtischer Flächen per Höchstgebotsverfahren. In diesem Kontext wurde auch die IBA Hamburg zur Entwicklung Wilhelmsburgs und der Veddel eingesetzt, um relativ zentral gelegene, aber ,problematische" städtische Räume zu vermarkten und diesen „weiße[n] Fleck auf der Landkarte“ (IBA o.D.) als Wohnort für urbane Mittelschichten ,zu entdecken“ und ,zu erobern‘. Festhalten lässt sich so, dass eine unternehmerische Orientierung seit Mitte der 1980er Jahre dominant und über Parteigrenzen hinweg in die stadtentwicklungspolitischen Rationalitäten vor allem auf der Senatsebene eingeschrieben wurde.[6]

\section{Die Konvergenz unternehmerischer und sozialer Stadtpolitik: Neue Wohnungspolitik als Strategie der Verbürgerlichung}

Fragen wir vor dem Hintergrund dieser genealogischen Rekonstruktion Hamburger Stadtentwicklungspolitik nun nach deren gegenwärtigen ReKonfigurationen, dann wird sichtbar, dass sozial-integrierte und unternehmerische Stadtpolitik, die sich als zwei parallele Strategien zur Bearbeitung städtischer Krisen herausgebildet haben, auf spezifische Weise 
ineinanderwirken. Wurden diese beiden Strategien in der bisherigen stadtpolitischen Debatte weitgehend als Gegensätze diskutiert (vgl. etwa Bauriedl 2007: 86), so werden durch eine genauere Analyse des Wohnungsbauprogramms, der Sozialen Erhaltungsverordnungen und der IBA Hamburg gewissermaßen ,synergetische“ Kooperationen sichtbar.

Zentral auffällig ist zunächst, dass alle diese Beispiele ihre ,soziale‘ Seite gerade in der Herstellung oder Bewahrung einer spezifischen sozialen Mischung haben: Das Neubauprogramm samt Drittelmix beinhaltet gewissermaßen eine präventive Mischungspolitik - explizit geht es darum, , homogene Neubauquartiere, die etwa ausschließlich aus Sozialwohnungen bestehen, zu verhindern (vgl. exemplarisch etwa zum, vorbildlich diversifizierten' Neubauprojekt „Mitte Altona“ Rinn 2016: 202). Beworben als Schaffung von Wohnraum ,für alle‘ bedeutet dieser Ansatz nun gerade nicht, dass auch ,alle‘ der sozial ungleichen Bewohner_innen potenziell Zugang zu allen dieser Neubauwohnungen bekommen, denn diese bestehen zu mehr als zwei Dritteln aus hochpreisigen Miet- oder Eigentumswohnungen.[7] Wenn gegenwärtig rund 80 Prozent aller Haushalte zur Miete wohnen, rund die Hälfte Anrecht auf eine Sozialwohnung hat (vgl. FHH 2017a), und nur noch 8,5 Prozent des Bestands Sozialwohnungen sind (FHH 2017c: 8), dann wird deutlich, dass die Strategie, Wohnungsmangel und steigende Mieten durch die Produktion vor allem teurer Miet- und Eigentumswohnungen zu bearbeiten, für Menschen mit geringen Einkommen und marginalisierten Positionen am Wohnungsmarkt keine Verbesserungen bringen wird. Das zeigen auch die fortgesetzten Mietsteigerungsdynamiken und das Schrumpfen von Wohnungsbeständen des unteren Preissegments.[8]

Die Sozialen Erhaltungsverordnungen dienen gerade der Stabilisierung des Status Quo in bereits gentrifizierten Gebieten. In den ehemaligen Sanierungsgebieten wurden sie zu einem Zeitpunkt erlassen, an dem politischadministrative Akteur_innen eine offenbar schützenswerte, funktionierende Mischung erkannten, sprich eine Mittelschichtsdominanz hergestellt wurde. Mit anderen Worten: Die Sozialen Erhaltungsverordnungen werden bislang in jenen Stadtteilen eingesetzt, in denen selbst schon Teile der Mittelschicht von Verdrängung bedroht sind. Hier wird aber gleichzeitig auch deutlich: Politiken sozialer Mischung funktionieren nicht nur in Richtung der De-Konzentration von Armut, sondern auch als Verhinderung einer weiteren stadträumlichen Segregation der oberen Mittel- und Oberschicht. Und so begrenzt die Wirkung der Sozialen Erhaltungsverordnungen de facto sein mag (vgl. Vogelpohl/Buchholz 2017, Vogelpohl 2013), so verweist der Ansatz der Investitionsbeschränkung in spezifischen Teilräumen doch auf eine übergreifend beobachtbare Strategie: Politisch-administrative Akteur_innen arbeiten daran, Investitionen und Mittelschichtsangehörige in diejenigen städtischen Räume zu lenken, in denen Aufwertung und die Neuzusammensetzung der Bewohner_innenschaft weiterhin erwünscht ist. Das ist gegenwärtig etwa der Hamburger Osten, der mit einem großformatigen Stadtentwicklungsprogramm aufgewertet und als Wohnstandort attraktiver gemacht werden soll (vgl. Metzger 2017), und das war zuvor vor allem Wilhelmsburg, dessen, Potenziale durch die IBA erschlossen und inwertgesetzt werden sollten.

Die IBA war zunächst eine großangelegte Marketing-Kampagne für einen Image- und Bevölkerungswandel (vgl. Hohenstatt/Rinn 2013; AKU 
Wilhelmsburg 2013). Langebevordieersteneue Wohnunggebaut wurde, wurde ein offensives rebranding Wilhelmsburgs vorangetrieben, etwa durch Kulturund Kreativpolitik und die (schon zuvor eingeführte) Subventionierung studentischen Wohnens und, innovativer Baugemeinschaften. Soziale Ungleichheit und Konflikte wurden in der IBA-Strategie weitgehend dethematisiert, stattdessen wurde positiv auf Diversität, Kultur und Bildung gesetzt. Die IBA hatte insofern aus den Widersprüchen des Soziale-Brennpunkte-Diskurses der gerade jene Quartiere diskursiv abwertete, die aufgewertet werden sollten - gelernt und setzte konsequent auf eine „festivalisierte Problembearbeitung“ (Hohenstatt/Rinn 2013). So war sie ein Stadtentwicklungsinstrument zur Angleichung von ,untergenutzten' Flächen und ,problematischen' Wohngebieten an immobilienwirtschaftliche Renditeerwartungen und Mittelschichtsnormen. Sozialer Wohnungsbau gehörte dabei, anders als das ,soziale“ Aushängeschild „Weltquartier“ suggerieren mag,[9] nie zu den Kernthemen der Bauausstellung. Die hier von Vogelpohl und Buchholz (2017) hervorgehobenen relativ langen Fristen der Sozialbindungen sind zwar bislang eine Ausnahme in Hamburg. Sie sind jedoch aus mindestens zwei Gründen kein progressives Beispiel: Erstens muss die Gesamtproblematik des sozialen Wohnungsbaus als temporäre „soziale Zwischennutzung“ (Holm, zitiert nach Metzger/Schipper 2017: 187) in Rechnung gestellt werden. Zweitens und noch wichtiger: Wilhelmsburg ist insgesamt ein „Freistellungsgebiet“ (vgl. FHH 2016a), die einkommensbezogenen Zugangsbeschränkungen zu Sozialwohnungen wurden aufgehoben, sodass dort auch Besserverdienende einziehen dürfen. Dies wurde explizit damit begründet, dass so die soziale Mischung verbessert werde. Betrachtet man die IBA insgesamt, so wurde das Versprechen einer ,Aufwertung ohne Verdrängung' systematisch nicht eingelöst - die Bauausstellung hatte dafür nicht nur schlicht keine Strategien, ihre mittelschichtsorientierte Attraktivierungspolitik hatte vielmehr den gegenteiligen Effekt.[10]

Die IBA ist möglicherweise das plastischste Beispiel der sich abzeichnenden Konvergenz unternehmerischer und sozialer Stadtentwicklungspolitik. In deren Aufwertungsstrategien werden gemeinsame normativ-strategische Konzeptionen einer ,guten Stadt für alle‘ produktiv: Das übergreifende Urbanitätsideal ist eine mittelschichtsaffine Nutzungs- und Bevölkerungsmischung, die über Attraktivierung beziehungsweise Stabilisierung städtischer Teilräume erreicht werden soll. Die Immobilienwirtschaft soll dabei eine mobilisierende Rolle spielen, aber auch ,sozial nachhaltig' reguliert und in entsprechende Bündnisse integriert werden, um die Probleme einer verwertungsorientierten Wohnraumversorgung weiterhin marktorientiert bearbeiten zu können. Die ,urbanen Mittelschichten' sollen für ,positive Quartierseffekte' sorgen, von denen alle - und gerade auch die ,benachteiligten' Bewohner_innen - profitieren sollen. Hier verbinden sich ökonomische Wachstumsstrategien und sozialpolitische Strategie gegen stadträumliche Benachteiligung und unerwünschte Segregation. Die Zielgruppen sozialer und unternehmerischer Stadtpolitik sind insofern teilweise identisch geworden. Zentrale Strategie in dieser unternehmerischintegrierten Rationalität ist eine Bevölkerungspolitik sozialer Mischung: eine spezifische räumliche An-Ordnung von (Un-)Gleichheit und Differenz der Stadtbewohner_innen, die auf die Normalisierung von Quartieren zielt. 
Orientierend für diese normativ-strategischen Mischungskonzeptionen sind dabei Visionen des gelingenden ,urbanen“ Zusammenlebens - Visionen, in denen es nicht um eine egalitäre Konzeption der ,Stadt für alle‘ geht und die zudem in ihrer bevölkerungspolitischen Realisierung autoritär werden. Die versprochene ,Stadt für alle soll als städtisches Mosaik diversifizierter, aber normalisierter ,funktionierender ' Teilräume entstehen, in denen die sozial ungleichen und kulturell verschiedenen Bewohner_innen die von ihnen erwarteten gesellschaftlichen Positionen und Handlungsrationalitäten beziehungsweise Verhaltensweisen annehmen sollen. Ziel ist es, möglichst überall durchaus unterschiedliche, aber kohäsive Stadträume zu schaffen, in denen eine heterogene Stadtbevölkerung sich so miteinander in Beziehung setzt, dass soziale Konflikte minimiert, ,Integrationsprozesse“ auch armer und marginalisierter Personengruppen organisiert und insgesamt eine Art sozialmoralischer trickle-down-Prozess in Gang gesetzt werden kann. Genau in dieser Version der ,guten Stadt für alle treffen sich die verschiedenen Strategien der Verbürgerlichung städtischer Räume.

\section{Schlussfolgerungen}

Betrachtet man also die jüngsten stadt- und wohnungspolitischen Re-Regulierungen in Hamburg vor dem Hintergrund der rekonstruierten Konvergenz sozialer und unternehmerischer Strategien, dann wird es problematisch, diese Re-Regulierungen als im positiven Sinne ,sozialer ${ }^{\text {zu }}$ begreifen, ihnen ,progressive“ Potenziale zuzuschreiben oder gar Brüche mit neoliberaler Politik zu identifizieren. Sie sind auch im bundesweiten Vergleich keine herausragenden Beispiele und lassen sich zudem, gemessen an den von Initiativen des Hamburger Netzwerks Recht auf Stadt artikulierten protagonistischen Ansprüchen und egalitären Forderungen nach einer ,Stadt für alle', nur schwer als ,Erfolge' sozialer Bewegungen begreifen. Vielmehr werden gerade in der veränderten Wohnungspolitik und dem vermehrten Einsatz sozialer Erhaltungsverordnungen (und auch den neuen Aufwertungsstrategien für ,Problemquartiere') Kontinuitäten und Verschiebungen einer bevölkerungspolitischen - und insofern auch ,neoliberalen'[11] - Regierung der Stadt sichtbar. Durch diese hindurch formiert sich ein Urbanismus der Ungleichheit, in dessen Konzeption einer ,Stadt für alle‘ soziale wie unternehmerische Stadtentwicklungsstrategien konvergieren und tendenziell ununterscheidbar werden. Soziale Integration in dieser ,Stadt für alle soll durch eine Strategie der Anreizung und Anrufung bürgerlicher Lebensweisen, aber auch der disziplinarischen Einordnung aller in mittelschichtsorientierte normative Ordnungen ermöglicht werden. Die Immobilienwirtschaft, entsprechend angereizt und geführt, soll ihren,Anteil an diesen Raumproduktionen haben, ohne dass ihre Verwertungsorientierung in Frage gestellt wird. Diese Arbeit an der Verbürgerlichung der Stadt will soziale Ungleichheiten nicht abbauen, sondern re-arrangieren. Das entsprechende soziale Programm bedeutet, dass auch den ,Benachteiligten“ ein Leben in einem mittelschichtsdominierten Quartier ermöglicht werden soll. In dieser ,Stadt für alle“ soll es langfristig keine Exklusion im Sinne von schlichter Verdrängung, problematischer' Bevölkerungsgruppen mehr geben - was nicht heißt, dass an den bürgerlichen normativen Raumordnungen 
nicht auch mit Kontrollstrategien und Ausschlüssen gegenüber den ,Unehrbaren', ,Kriminellen', und ,gefährlichen“ Anderen gearbeitet wird (vgl. Rinn 2017). Es sollen jedoch zumindest programmatisch weder Räume für die ,Ausgeschlossenen', möglichst peripher und abgeschlossen von der bürgerlichen Stadt, entstehen, noch abgeschottete Räume der ,Wohlhabenden und,Wohlanständigen'. Hier werden auch zentrale Widersprüche der unternehmerisch-integrierten Stadtentwicklungsrationalität sichtbar. Wohnen ,für alle zu ermöglichen und Verdrängung zu verhindern entspricht zwar den normativen Selbstpositionierungen politisch-administrativer Akteur_innen, jedoch: Ihre Konzeption der, guten Stadt für alle' atmet den Urbanismus der Ungleichheit, und in diesem ist Verdrängung im Interesse, funktionierender sozialer Mischung kein bedauerlicher Nebeneffekt, sondern zentraler strategischer Einsatz.

\section{Endnoten}

[1] Meine empirischen Grundlagen sind Dokumentenanalysen, Expert_inneninterviews und teilnehmende Beobachtungen, mittels derer ich Rationalitäten und Praktiken von Akteur_innen rekonstruiert habe, die an Konflikten um Stadtentwicklungspolitik in Hamburg (im Kontext des Recht auf Stadt-Netzwerks seit 2009) beteiligt waren (vgl. Rinn 2016). Die Analysen zur IBA Hamburg stehen zugleich mit meiner Mitarbeit im Arbeitskreis Umstrukturierung Wilhelmsburg in Zusammenhang. Ich danke Lisa Carstensen, Joscha Metzger, Jan Wehrheim, den beiden Gutachter_innen sowie der Redaktion für ihre hilfreichen kritischen Kommentare.

[2] In Hamburg sind drei Viertel aller Wohnungen Mietwohnungen (vgl. Vogelpohl/Buchholz 2017). Zwischen 2000 und 2011 stiegen Angebotsmieten durchschnittlich um 38 Prozent auf 11,21 Euro (alle Angaben pro $\mathrm{m}^{2}$, netto/kalt), die höchsten Steigerungsraten waren in innenstadtnahen Stadtteilen Hohenfelde (76 Prozent auf 1o Euro), St. Georg (72 Prozent auf 13 Euro) oder Altona-Nord/Altona-Altstadt (63 Prozent auf 13,02 Euro), aber auch St. Pauli (51 Prozent auf 11,84 Euro) zu verzeichnen (vgl. Pohl/Wischmann 2014: 48). Zur Einordnung: Die verfügbaren Daten zur Lohnentwicklung in Hamburg zeigen eine nur 14-prozentige Steigerung der Bruttolöhne an (vgl. FHH 2017c: 12).

[3] Mittlerweile wurde der Anteil geförderter Wohnungen am Gesamtneubauvolumen leicht auf 30 Prozent gesenkt.

[4] Verbürgerlichung verweist zum einen auf embourgeoisment, womit in der Gentrifizierungsdebatte die klassenpolitische Dimension von Aufwertung und Verdrängung betont wird (vgl. Slater 2006), zum anderen auf Politiken, die auf die Hervorbringung von sozial integrierten, durch ,Bürgerlichkeit‘ geprägten städtischen Räumen abzielen. Bürgerlichkeit wird, wie Lanz (2013) in seiner Analyse urbaner Regierungsweisen in Berlin herausgearbeitet hat, in ihren ökonomischen, politischen und kulturellen Dimensionen im Kontext einer „Regierung durch citizenship“ (ebd.: 1309ff.) produktiv, die mit sozialen, quartiersorientierten Problembearbeitungsstrategien korrespondiert. Mit Verbürgerlichung bezeichne ich daher nicht einfach Bevölkerungsaustausch oder wohnraumbezogene Verdrängung, sondern Strategien, die gerade via Politiken urbaner diversity und sozialer Mischung (sozial integrierte und ökonomisch erfolgreiche) Quartiere hervorzubringen versuchen - Quartiere, in denen westlich-kulturelle, ökonomischunternehmerische und politisch-gemeinwohlorientierte bürgerliche Normvorstellungen und Praktiken dominieren, die zugleich auch für eine disziplinierende Integration der ,Anderen' der bürgerlichen Klassen sorgen sollen.

[5] In solchen Desintegrationsdiagnosen und Anrufungen urbaner Bürgerlichkeit werden auch kulturell-rassistische Problematisierung (etwa von ,Parallelgesellschaften') produktiv (vgl. Lanz 2013). Die Figur der bürgerlichen (Mittelschichts-)Familie (wie pluralisiert und modernisiert auch immer) wird dabei noch immer als Garantin für stadtgesellschaftliche Stabilität begriffen und ist zudem zunehmend zur Adressatin von Standortpolitik im Metropolenwettbewerb geworden (vgl. Frank 2014). 
[6] Nicht wenige der stadtentwicklungspolitischen Projekte des Unternehmens Hamburg scheiterten jedoch am Widerstand der Bewohner_innenschaft: etwa die Neubebauung des Elbhangs von St. Pauli (verhindert durch die Hafenstraßenbesetzung ab 1981, vgl. Borgstede 2010, und den „Park Fiction“ ab 1994), das Musicalhaus „Neue Flora“ im Schanzenviertel (seit 1989 als „Rote Flora“ besetzt, vgl. Birke 2014), und nicht zuletzt 2003 und 2015 Bewerbungen für Olympischen Sommerspiele.

[7] 2016 waren 31,5 Prozent der Neubauwohnungen öffentlich gefördert (vgl. FHH 2017a), 2015 und 2014 wurden die Zielzahlen mit 25 und 29 Prozent noch deutlicher unterschritten (vgl. FHH 2016b).

[8] Zwischen 2011 und 2017 stiegen die durchschnittlichen Angebotsmieten um weitere 13 Prozent auf 12,69 Euro (vgl. Gymnasium Ohmoor 2017). Ende 2016 konnte noch immer über 9.000 als „vordringlich wohnungssuchend“ anerkannten Haushalten keine Wohnung vermittelt werden (vgl. FHH 2017b: 8). Zwischen 2010 und 2017 hat sich der Sozialwohnungsbestand im 1. Förderweg um 22 Prozent auf knapp 79.ooo Wohnungen reduziert (vgl. FHH 2017c: 8). Dieser anhaltende Trend (vgl. Metzger/Schipper 2017: 195) kann allein durch Wohnungsneubau kaum gestoppt werden.

[9] Zu den Konflikten um Sanierung, Mietsteigerungen (die es trotz öffentlicher Förderungen gab) und Verdrängung im „Weltquartier“ vgl. Birke/Hohenstatt/Rinn 2015, AKU Wilhelmsburg 2013. Bemerkenswert ist, dass weder die IBA noch die städtische SAGA als Eigentümerin ihr Versprechen, niemanden aus dem „Weltquartier“ zu verdrängen, systematisch dokumentiert hat. So gibt es keine Begleitforschung zu den Gründen, warum von den 687 im Sanierungsprozess umquartieren Mietparteien 460 nicht zurück ins Quartier zogen (vgl. FHH 2016c). In einer Auskunft des Senats heißt es lapidar, viele seien „aus persönlichen Gründen nicht in das Quartier zurückgezogen“ (vgl. ebd.). Das mag für Sanierungsprojekte dieses Formats nicht untypisch sein, wäre aber angesichts des beanspruchten ,Modellcharakters' doch einer genaueren Untersuchung wert gewesen.

[10] Die IBA Hamburg GmbH selbst wehrte sich gegen den Gentrifizierungsvorwurf unter anderem mit dem Argument, dass Mietsteigerungen in Wilhelmsburg mit 40 Prozent zwischen 2006 und 2013 im Hamburger Durchschnitt lägen (vgl. IBA Hamburg 2013). Damit hat sich der Stadtteil unter der IBA zumindest ,normalisiert', wobei über kleinräumige Steigerungsdynamiken innerhalb des sehr heterogenen Gebiets keine Daten vorliegen.

[11] Bevölkerungspolitik, die ,mit dem Markt regiert', kann als einer der Kerne neoliberaler Gouvernementalität begriffen werden (vgl. Foucault 2004).

\section{Autor_innen}

Moritz Rinn ist Politikwissenschaftler, seine Arbeitsschwerpunkte sind städtische Konflikte, Stadtentwicklungspolitik, Politiken des Sozialen und Sozialstaatlichkeit.

moritz.rinn@uni-due.de

Die Publikation dieses Beitrags wurde durch den Open-Access-Fonds der Universität Duisburg-Essen ermöglicht.

\section{Literatur}

AKU Wilhelmsburg (2013): Unternehmen Wilhelmsburg. Stadtentwicklung im Zeichen von IBA und igs. Berlin/Hamburg: Assoziation A.

Alisch, Monika / Dangschat, Jens S. (1998): Armut und soziale Integration. Strategien sozialer Stadtentwicklung und lokaler Nachhaltigkeit. Opladen: Leske + Budrich.

Bauriedl, Sybille (2007): Spielräume nachhaltiger Entwicklung. Die Macht stadtentwicklungspolitischer Diskurse. München: oekom.

Birke, Peter (2014): Autonome Sehenswürdigkeit. Die Rote Flora und die Hamburger Stadtentwicklung seit den späten 1980er Jahren: In: Sozial.Geschichte Online. Zeitschrift für historische Analyse des 20. und 21. Jahrhunderts 6/13, 80-104. 
Birke, Peter (2010): Herrscht hier Banko? Die aktuellen Proteste gegen das Unternehmen Hamburg. In: Sozial.Geschichte Online. Zeitschrift für historische Analyse des 20. und 21. Jahrhunderts 2/3, 148-191.

Birke, Peter / Hohenstatt, Florian / Rinn, Moritz (2015): Gentrification, social action and „role-playing“: Experiences garnered on the outskirts of Hamburg. In: International Journal of Action Research 11/1-2, 195-227.

Borgstede, Simone Beate (2010): Der Kampf um die Herzen und Köpfe der Menschen. St. Pauli Hafenstraße, 1981 bis 1987. In: Das Argument 52/289, 849-858.

Breckner, Ingrid (2013): Urban poverty and gentrification. A comparative view on different areas in Hamburg. In: Hans-Christian Petersen (Hg.), Spaces of the Poor. Perspectives of Cultural Sciences on Urban Slum Areas and Their Inhabitants. Bielefeld: transcript, 193-207.

Dangschat, Jens S. (1993): Konzeption, Realität und Funktion ,neuer Standortpolitik ${ }^{\star}$ - am Beispiel des 'Unternehmens Hamburg'. In: Hubert Heinelt / Margit Mayer (Hg.), Politik in europäischen Städten. Basel/Boston/Berlin: Birkhäuser, 29-48.

Demirović, Alex (2013): Kritik der Politik. In: Rahel Jaeggi / Daniel Loick (Hg.), Nach Marx. Philosophie, Kritik, Praxis. Berlin: Suhrkamp, 463-485.

Dohnanyi, Klaus von (1983): „Unternehmen Hamburg“. http://uebersee-club.de/resources/ Server/pdf-Dateien/1980-1984/vortrag-1983-11-29Dr.\%20Klaus\%20von\%2oDohnanyi. pdf (letzter Zugriff am 11.1.2018).

Eckardt, Frank (2017): Architecture and the ,Right to the City': The IBA Hamburg as a case for critical urban studies. In: Maria Manuela Mendes / Teresa Sá / João Cabral (Hg.), Architecture and the Social Sciences. Cham: Springer, 121-135.

FHH (2017a): Freie und Hansestadt Hamburg, Bürgerschaft: Drucksache 21/8286, 17.03.2017. Hamburg.

FHH (2017b): Freie und Hansestadt Hamburg, Bürgerschaft: Drucksache 21/9012, 02.06.2017. Hamburg.

FHH (2017c): Freie und Hansestadt Hamburg, Bürgerschaft: Drucksache 21/9491, 14.07.2017. Hamburg.

FHH (2017d): Freie und Hansestadt Hamburg, Bürgerschaft: Drucksache 21/11139, 28.11.2017. Hamburg.

FHH (2016a): Freie und Hansestadt Hamburg, Bürgerschaft: Drucksache 21/5213, 19.07.2016. Hamburg.

FHH (2016b): Freie und Hansestadt Hamburg, Behörde für Stadtentwicklung und Wohnen: Wohnungsbaubericht Hamburg 2014/2015. Hamburg.

FHH (2016c): Freie und Hansestadt Hamburg, Bürgerschaft: Drucksache 21/6769, 25.11.2017. Hamburg.

FHH (2002): Freie und Hansestadt Hamburg, Staatliche Pressestelle: Leitbild: Metropole Hamburg - Wachsende Stadt; http://epub.sub.uni-hamburg.de/epub/ volltexte/2008/2282/pdf/data.pdf (letzter Zugriff am 12.4.2018).

FHH (1980): Freie und Hansestadt Hamburg: Stadtentwicklungskonzept. Beschlossen vom Senat der Freien und Hansestadt Hamburg am 30. September 1980. Hamburg.

Foucault, Michel (2004): Geschichte der Gouvernementalität I: Sicherheit, Territorium, Bevölkerung. Frankfurt am Main: Suhrkamp.

Fraeser, Nina (2017): ,Fantasies of antithesis'. Assessing Hamburg's Gängeviertel as a tourist attraction. In: Claire Colomb / Johannes Novy (Hg.), Protest and Resistance to the Tourist City. London/New York: Routledge, 320-339.

Frank, Susanne (2014): Mittelschichtfamilien als Adressaten und Motoren der Stadt- und Quartiersentwicklung. In: Informationen zur Raumentwicklung 41/4, 361-373.

Frank, Susanne (2003): Stadtplanung im Geschlechterkampf. Stadt und Geschlecht in der Großstadtentwicklung des 19. und 20. Jahrhunderts. Opladen: Leske + Budrich.

Füllner, Jonas / Templin, David (2011): Stadtplanung von unten. Die „Recht auf Stadt“Bewegung in Hamburg. In: Andrej Holm / Dirk Gebhard (Hg.), Initiativen für ein Recht auf Stadt. Theorie und Praxis städtischer Aneignung. Hamburg: VSA, 79-104.

Güntner, Simon (2013): Kalkulierbare Segregation? Drei Perspektiven aufdiesozialräumliche Polarisierung in Hamburg. In: Gerd Pohl / Klaus Wicher (Hg.), Hamburg: gespaltene Stadt? Soziale Entwicklungen in der Metropole. Hamburg: VSA, 34-53.

Güntner, Simon (2007): Soziale Stadtpolitik. Institutionen, Netzwerke und Diskurse in der Politikgestaltung. Bielefeld: transcript. 
Gymnasium Ohmoor (2017): Preisentwicklung auf dem freien Mietwohnungsmarkt in Hamburg. http://www.bautschweb.de/mieten/Preisentwicklung.gif (letzter Zugriff am 11.1.2018).

Häußermann, Hartmut / Läpple, Dieter / Siebel, Walter (2008): Stadtpolitik. Frankfurt am Main: Suhrkamp Verlag.

Hohenstatt, Florian / Rinn, Moritz (2013): Festivalisierte Problembearbeitung. Die bevölkerungspolitische Strategie der IBA Hamburg, die Abwesenheit sozialer Arbeit in der Stadtentwicklungspolitik und die Effekte auf Wohnverhältnisse in Wilhelmsburg. In: Widersprüche. Zeitschrift für sozialistische Politik im Bildungs-, Gesundheits- und Sozialbereich 33/127, 23-38.

Holm, Andrej (2017): Mehr Licht als Schatten. Berliner Wohnungspolitik in Rot-Rot-Grün. In: dérive - Zeitschrift für Stadtforschung 68, 14-17.

Holm, Andrej (2014). Feigenblatt Mietpreisbremse. In: Blätter für deutsche und internationale Politik 5, 20-22.

Holm, Andrej (2006): Die Restrukturierung des Raumes. Stadterneuerung der 9oer Jahre in Ostberlin: Interessen und Machtverhältnisse. Bielefeld: transcript.

Huning, Sandra / Schuster, Nina (2015): ,Social Mixing“ or ,Gentrification“? Contradictory perspectives on urban changes in the Berlin district of Neukölln. In: International Journal of Urban and Regional Research 39/4, 738-755.

IBA Hamburg (2013): IBA Strukturmonitoring 2013. Endbericht. Hamburg.

IBA Hamburg (o.D.): Das Projektgebiet. http://www.iba-hamburg.de/story/ das-projektgebiet.html (letzter Zugriff am 11.1.2018).

Jessop, Bob (2007): State Power. A Strategic-Relational Approach. Cambridge/Malden: Polity Press.

Jessop, Bob (2002): The Future of the Capitalist State. Cambridge/Malden: Polity Press.

Kaltenbrunner, Robert / Waltersbacher, Matthias (2014): Besonderheiten und Perspektiven der Wohnsituation in Deutschland. In: Aus Politik und Zeitgeschichte 20-21, 3-12.

Lanz, Stephan (2013): Be Berlin! Governing the city through freedom. In: International Journal of Urban and Regional Research 37/4, 1305-1324.

Lees, Loretta (2010): Planning urbanity? In: Environment and Planning 41/10, 2302-2308.

Lessenich, Stephan (2008): Die Neuerfindung des Sozialen. Bielefeld: transcript.

Maier, Willfried (1999): Politik der Stadterneuerung als soziale Aufgabe. In: FritzSchumacher-Kolloquium e.V. (Hg.), „Stadterhaltung - Stadtumbau - Stadterneuerung“. Strategien zur Steuerung des städtischen Wandels. Hamburg: Sautter + Lackmann, 12-15.

Mayer, Margit (2002): Soziales Kapital und Stadtentwicklungspolitik - ein ambivalenter Diskurs. In: Michael Haus (Hg.), Bürgergesellschaft, soziales Kapital und lokale Politik: Theoretische Analysen und empirische Befunde. Wiesbaden: VS Verlag für Sozialwissenschaften, 33-58.

Metzger, Joscha / Schipper, Sebastian (2017): Postneoliberale Strategien für bezahlbaren Wohnraum? Aktuelle wohnungspolitische Ansätze in Frankfurt am Main und Hamburg. In: Barbara Schönig / Justin Kadi / Sebastian Schipper (Hg.), Wohnraum für alle?! Perspektiven auf Planung, Politik und Architektur. Bielefeld: transcript, 181-212.

Metzger, Joscha (2017): Partizipation in der unternehmerischen Stadt? Quartiersentwicklung im Hamburger Osten aus der Perspektive der Bewohner_innen. In: Patrick Oehler / Nadine Käser / Matthias Drilling / Jutta Guhl / Nicola Thomas (Hg.), Emanzipation, Soziale Arbeit und Stadtentwicklung. Eine programmatische und methodische Herausforderung. Opladen: Barbara Budrich, 277-295.

Peddinghaus, Pia / Hauer, Dirk (1998): Der Sozialstaat zeigt seine Zähne. Sozialpolitik und Ausgrenzungsstrategien in Hamburg. In: StadtRat (Hg.), Umkämpfte Räume. Hamburg/ Berlin/Göttingen: Verlag Libertäre Assoziation/Verlag der Buchläden Schwarze Risse Rote Strasse, 103-119.

Pohl, Thomas / Wischmann, Katharina (2014): Wohnungsmarktdynamikund stadtpolitische Konflikte in Hamburg. Ein Beitrag zur Gentrificationforschung. In: Europa Regional 19/2, 41-55.

Polkowski, Dieter (2001): Städtebauförderung als Instrument einer nachhaltigen Stadtentwicklung. Das Beispiel Hamburg. In: Informationen zur Raumentwicklung 9-10, 597-595.

Rinn, Moritz (2017): Verdrängung in der unternehmerischen Stadt. Zum Beispiel Hamburg-St. Georg. In: FORUM für Kinder- und Jugendarbeit 33/3, 4-9. 
Rinn, Moritz (2016): Konflikte um die Stadt für alle. Das Machtfeld der Stadtentwicklungspolitik in Hamburg. Münster: Verlag Westfälisches Dampfboot.

Schönig, Barbara/Rink, Dieter/Gardemin, Daniel/Holm,Andrej(2017): Paradigmenwechsel in der kommunalen Wohnungspolitik? Variationen kommunalisierter Wohnungspolitik im transformierten Wohlfahrtsstaat. In: Marlon Barbehön / Sybille Münch (Hg.), Variationen des Städtischen - Variationen lokaler Politik. Wiesbaden: Springer VS, 25-62.

Schubert, Dirk (2011): Gleichzeitigkeiten von Leerstand und neuer Wohnungsnot. In: Widersprüche. Zeitschrift für sozialistische Politik im Bildungs-, Gesundheits- und Sozialbereich 31/121, 23-36.

Slater, Tom (2006): The eviction of critical perspectives from gentrification research. In: International Journal of Urban and Regional Research 30/4, 737-757.

Vogelpohl, Anne (2013): Mit der Sozialen Erhaltungssatzung Verdrängung verhindern? Zur gesetzlichen Regulation von Aufwertungsprozessen am Beispiel Hamburg. Arbeitspapier. https://www.geo.uni-hamburg.de/geographie/dokumente/personen/publikationen/ vogelpohl/vogelpohl_soziale-erhaltungssatzung.pdf (letzter Zugriff am 12.4.2018).

Vogelpohl, Anne / Buchholz, Tino (2017): Breaking with neoliberalization by restricting the housing market: Novel urban policies and the case of Hamburg. In: International Journal of Urban and Regional Research 41/2, 266-281.

Vogelpohl, Anne / Vollmer, Lisa / Vittu, Elodie / Brecht, Norma (2017): Die Repolitisierung des Wohnens. Städtische soziale Bewegungen für ein Recht auf Wohnen und auf Stadt in Hamburg, Berlin, Jena und Leipzig. In: Barbara Schönig / Justin Kadi / Sebastian Schipper (Hg.), Wohnraum für alle?! Perspektiven auf Planung, Politik und Architektur. Bielefeld: transcipt, 105-130.

Vrenegor, Nicole (2012): Entlang einer imaginären Linie. Drei Jahre Recht auf StadtBewegung in Hamburg - ein Zwischenstopp. In: derive - Zeitschrift für Stadtforschung 49, 9-14.

\section{An Urbanism of Inequality. ,New social urban politics' in Hamburg as strategies of embourgeoisment.}

In Germany, the housing question is back on the agenda. Various political reregulations could be observed, aiming at, affordable housing for all'. Now, researchers discuss whether these, social're-regulations indicate an end of urban neoliberalization. I contribute to this debate by analyzing urban policy in Hamburg. Is Hamburg an example for ,progressive“ strategies towards a more just ,city for all', as scholars have recently argued? To answer this question, I first discuss the current urban policy in comparison to other cities in Germany. I then adopt a genealogical perspective on the specific rationalities which shape the current, new social urban policy'. Against this backdrop, I re-interpret the current re-regulations. One central finding is, that they are less, progressive' or ,post-neoliberal', but manifestations of an ,urbanism of inequality'. The ,city for all'shall be realized through strategies of ,embourgeoisment' which foster inequality rather than reducing it. 
